\title{
Effects of Chitosan on the Secretion of Cytokines and Expression of Inducible Nitric Oxide Synthase mRNA in Peritoneal Macrophages of Broiler Chicken
}

\author{
Huiying $\mathrm{Li}^{1,2}$, Binlin Shi ${ }^{1 *}$, Sumei Yan ${ }^{1}$, Tianzhang $\mathrm{Zhao}^{2}$, Junliang $\mathrm{Li}^{1}$ and Xiaoyu Guo ${ }^{1}$ \\ ${ }^{l}$ College of Animal Science; Inner Mongolia Agricultural University; Huhhot - P. R. China. ${ }^{2}$ Department of \\ Biotechnology; Wulanchabu Vocational College; Wulanchabu - P. R. China
}

\begin{abstract}
An in vitro experiment was conducted to study the effects of chitosan on the secretion of cytokines and expression of inducible nitric oxide synthase mRNA in peritoneal macrophages of broiler chicken. In the experiment, peritoneal macrophages were incubated for $24 \mathrm{~h}$ in culture medium supplemented with 0 (control), 40, 80, 160 and $320 \mu \mathrm{g} / \mathrm{mL}$ chitosan. The results showed that chitosan tended to increase quadratically the levels of interleukin-1 $(P=0.093)$ and interleukin-2 $(P=0.106)$ in the culture fluid of peritoneal macrophage. Chitosan also significantly enhanced inducible nitric oxide synthase mRNA expression of peritoneal macrophage in a quadratic dose-dependent manner $(P<0.05)$ and tended to promote quadratically the secretion of nitric oxide $(P=0.053)$ and inducible nitric oxide synthase $(P=0.157)$ in peritoneal macrophages. This result implied that one of the mechanisms by which chitosan modulated immune functions in chickens might be chitosan activating expression of inducible nitric oxide synthase and then improving the secretion of nitric oxide.
\end{abstract}

Key words: Chitosan, Broiler chicken, Peritoneal macrophages, Cytokine, Inducible nitric oxide synthase

\section{INTRODUCTION}

Chitosan, deacetylated chitin and natural alkaline polysaccharide with positive charges derived from the crabs, shrimps, insects, and other marine creatures, has been shown to promote the growth and immune functions of the animals (Shi et al. 2005). It has been reported that chitosan has a significant enhancing effect on the cellular and humoral immune function (Nishimura et al. 1986), and could stimulate macrophages in the rats and increase the NO, IL-1 and TNF- $\alpha$ secretion (Peluso et al. 1994). Several in vitro studies have shown that polysaccharide bound to the specific receptors on the surface of macrophage switched on signal transduction, thereby affecting the gene expression in macrophages and regulating immune function (Jamas et al. 1997).

Macrophages are special phagocytes that act as the immune effector cells, which participate in the regulation of immune extensively. They are derived from the monocytes, which are originally produced from the pluripotent stem cells in the bone marrow (Andus et al. 1995). The macrophages, acting in the innate immunity, through the destruction of pathogens agents and adaptative antigens presentation are an important defense mechanism of the birds (Qureshi 2003). Activated macrophages can secrete cytokines and mediators of inflammation, including interferon- $\gamma$ $(\mathrm{IFN}-\gamma)$, tumor necrosis factor- $\alpha$ (TNF- $\alpha$ ), interleukin-1 (IL-1), interleukin-2 (IL-2),

*Author for correspondence: shibinlin@eyou.com 
interleukin-6 (IL-6), macrophage colony stimulating factor (M-CSF), nitric oxide (NO). These mediators may increase the vascular permeability and are favorable to accumulation and infiltration of immune molecules and cells (Liu et al. 1993). TNF- $\alpha$ is known to be a key mediator for the induction of apoptosis and development of humoral immune response. Cytokines (IL-1 $\beta$, IL-2) play a central role in the cell-mediated immune response and also participate in the maintenance of tissue integrity ( $\mathrm{Li}$ et al. 2007). NO is a highly reactive signaling molecule and inflammatory mediator from Larginine by inducible nitric oxide synthase (iNOS), which modulates the immune responses and inflammation (Moilanen et al. 1999; Korhonen et al. 2005). iNOS is responsible for generating high levels of $\mathrm{NO}$ in activated macrophages, and its expression is essential for the functions of NO in the regulation of immune responses (Xing and Schat 2000). Porporatto et al. (2003) found that chitosan significantly enhanced the NO content and iNOS expression in rat macrophages. This implied that enhanced immune functions by chitosan are associated with the activation and strengthening of secretory functions of macrophages and increased expression of iNOS. Previous studies have shown that chitosan improved NO content and iNOS activity in serum as well as relative expression of iNOS mRNA in the small intestine of broiler chickens ( $\mathrm{Li}$ et al. 2009). However, there are very few data in this area, especially about the molecular biologic mechanisms, by which chitosan modulates the peritoneal macrophage of broiler chickens.

The objective of the present study was to explore the effects of chitosan on the secretion of cytokines and expression of iNOS mRNA in peritoneal macrophage of broiler chickens in vitro and to investigate the probable mechanism by which chitosan modulated the immune functions of chickens.

\section{MATERIALS AND METHODS}

\section{Chitosan}

The protocol of the present experiment was approved by the Animal Care and Use Committee, Inner Mongolia Agricultural University, Huhhot, China. The chitosan used in this study was provided by the Jinan Haidebei Marine Bioengineering Co. Ltd. (Shandong Province,
China) and its degree of de-acetylation was $90.52 \%$. Stock solutions of chitosan were prepared in $0.5 \%$ acetic acid and diluted with RPMI-1640 to the appropriate concentration for the experiments.

\section{Isolation and Purification of Peritoneal Macrophages}

Twenty-one day old Arbor Acre male broiler chicken was used. Peritoneal cells were harvested by the sterile lavage with $20 \mathrm{~mL}$ RPMI-1640 (Gibco, USA) medium without fetal calf serum. Macrophages were purified by adherence onto 24well flat-bottomed tissue culture plates in complete RPMI-1640 medium supplemented with $10 \%$ (v:v) heat-inactivated fetal calf serum (TBD, China), $100 \mathrm{U} / \mathrm{mL}$ of penicillin, $100 \mu \mathrm{g} / \mathrm{mL}$ of streptomycin, and $25 \mathrm{mmol} / \mathrm{L}$ HEPES buffer (Sigma, USA). Nonadherent cells were removed after $2 \mathrm{~h}$ at $37^{\circ} \mathrm{C}$. Cells were then counted on a hemocytometer using a light microscope and the viability was determined by a trypan blue exclusion method.

\section{Cell Culture}

Cell suspensions at a density of $1 \times 10^{6}$ cells $/ \mathrm{mL}$ culture medium were incubated in a 24 -well plate ( $1 \mathrm{~mL} /$ well) containing $0,40,80,160$, and 320 $\mu \mathrm{g} / \mathrm{mL}$ chitosan, with nine replicates in each treatment. After $24 \mathrm{~h}$ of incubation at $37^{\circ} \mathrm{C}$ in a $5 \% \mathrm{CO}_{2}$ humidified atmosphere, supernatants were collected after centrifugation $\left(800 \times \mathrm{g}\right.$ at $4^{\circ} \mathrm{C}$ for 10 $\mathrm{min}$ ) and stored at $-80^{\circ} \mathrm{C}$ for the analysis of IL-1, IL-2, TNF- $\alpha$, NO, and iNOS. The cells were harvested and stored at $-80^{\circ} \mathrm{C}$ to isolate the total RNA.

\section{Determination of Cytokines}

The levels of IL-1, IL-2 and TNF- $\alpha$ in the culture liquid of peritoneal macrophage were measured by radioimimunoassay according to the manufacturer's instructions (Zhang et al. 2008). The kits of the three cytokines were produced by Beijing Huaying Institute of Biotechnology and the detecting instrument was GC-911- $\gamma$ radioimmunity arithmometer.

\section{Determination of NO and iNOS Levels}

The levels of NO and iNOS in the culture liquid of peritoneal macrophages were analyzed by the absorption spectrometry using commercially available NO kits and iNOS kits (Nanjing Jiancheng Institute of Bioengineering) according 
to the manufacturer's instructions (Ding et al. 1988; Wang et al. 1995).

\section{RNA Extraction and Reverse Transcription (RT)}

RNA was extracted from the peritoneal macrophages using the RNA Fast 200 kits (Shanghai Feijie Biotech. Co. Ltd. China) according to the manufacturer's instructions. All the steps were carried out under RNase-free conditions. RNA integrity was verified electrophoretically by ethidium bromide staining and its purity was determined using UV-clear Microplates (TECAN) at $\mathrm{OD}_{260}$ and $\mathrm{OD}_{280}$. The $\mathrm{OD}_{260} / \mathrm{OD}_{280}$ ratio of all the samples was above 1.80. The RNA yield from the samples was too low to be accurately quantified by spectrometry, hence, $6.5 \mu \mathrm{L}$ RNA aliquots were amplified. All the RNA was treated with RNase-free DNase I to remove any possible genomic DNA contamination. For amplification of the targets, RT and PCR were run in two separate steps. Total RNA was reverse transcribed into cDNA using PrimeScriptTM RT reagent kits (TaKaRa, Inc. Dalian, China), following the manufacturer's directions. RT reaction parameters were as follows: RT at $37^{\circ} \mathrm{C}$ for $15 \mathrm{~min}$, RT inactivation at $85^{\circ} \mathrm{C}$ for $5 \mathrm{sec}$. The RT reaction mixture $(10 \mu \mathrm{L})$ contained $0.5 \mu \mathrm{L}$ PrimeScriptTM RT Enzyme Mix I, $2 \mu \mathrm{L} 5 \times$ PrimeScript buffer, $0.5 \mu \mathrm{L}$ Random 6 mers and $0.5 \mu \mathrm{L}$ Oligo dT Primer. The RT products (cDNA) were stored at $-20^{\circ} \mathrm{C}$ pending quantitative PCR assay.

\section{Real-time PCR for Quantification Of iNOS mRNA}

Primers used in this study were as follows: $\beta$ Actin (118 bp, GenBank accession no. NM_205518) $5^{\prime}-$ GCCAACAGAGAGAAGATGACAC-3'

(forward) and 5'-GTAACACCATC
ACCAGAGTCCA-3' (reverse); iNOS (371bp, GenBank accession no. U46504) 5'-AGGCCA AACATCCTGGAGGTC-3' (forward) and 5'TCATAGAGA CGCTGCTG CCAG-3' (reverse). The threshold cycle $(\mathrm{Ct})$ value represents the cycle number at which sample fluorescence rises statistically above the back-ground. Relative levels of iNOS mRNA were quantified using SYBR ${ }^{\circledR}$ PrimeScriptTM RT-PCR kits (TaKaRa, Inc. Dalian, China) following the manufacturer's instructions and a DNA Engine Opticon 2 fluorescence detection system (MJ research, USA) according to optimized PCR protocols. Reactions were also performed with negative controls (water replacing the cDNA). The PCR reaction system $(20 \mu \mathrm{L})$ contained $10 \mu \mathrm{L} 2 \times \mathrm{SYBR} \circledast$ Premix Ex TaqTM and $0.4 \mu \mathrm{L}(10 \mu \mathrm{M})$ of forward and reverse specific primers, $2 \mu \mathrm{L}$ of cDNA template and 7.2 $\mu \mathrm{L}$ RNA-free $\mathrm{H}_{2} \mathrm{O}$. The same dilution was used for both the iNOS and $\beta$-Actin. For the PCR reaction, the following experimental protocol was used: one cycle at $95^{\circ} \mathrm{C}$ for $1 \mathrm{~min}$; followed by 45 cycles, each cycle consisted of $5 \mathrm{sec}$ at $95^{\circ} \mathrm{C}$ for denaturation, $30 \mathrm{sec}$ at $62^{\circ} \mathrm{C}$ for annealing, and 10 sec at $72^{\circ} \mathrm{C}$ for extension; a final extension at $72^{\circ} \mathrm{C}$ for $7 \mathrm{~min}$; and melting curve program between $70^{\circ} \mathrm{C}$ and $95^{\circ} \mathrm{C}$ with a heating rate of $0.5^{\circ} \mathrm{C} / \mathrm{s}$ and a continuous fluorescence measurement. Fluorescence data were acquired after the extension step during the PCR reactions that contained SYBR Green. Thereafter, PCR products were analyzed by generating a melting curve. The melting curve of a product is sequence-specific and can be used to distinguish the non-specific from specific PCR products. Real time PCR efficiencies of iNOS and $\beta$-Actin were acquired by amplification of a dilution series of PCR products and were close to one. Expression level of iNOS was calculated as relative values using the $2^{-\Delta \mathrm{Ct}}$ method, where $\Delta \mathrm{Ct}$ was equal to $\mathrm{Ct}$ of the iNOS minus Ct of the $\beta$-Actin (Tropea et al. 2007). The sizes of RT-PCR products were confirmed by $2 \%$ agarose gel electrophoresis in the presence of ethidium bromide and bands were visualized by the exposure to ultraviolet light. Sequences were confirmed by the Sangon Biological Engineering Technology and Services Co. Ltd. (Shanghai, China).

\section{Statistical Analysis}

All data were subjected to General Linear Model procedure of SAS software (SAS Institute, 1998). Regression analysis was conducted to evaluate the linear and quadratic effects of chitosan on the various response criteria. A level of $p<0.05$ was used as the criterion for statistical significance.

\section{RESULTS}

The levels of IL-1, IL-2 and TNF- $\alpha$ in the culture fluid of peritoneal macrophage containing different concentrations of chitosan were higher than the control, except for IL-1 level in the treatment of $320 \mu \mathrm{g} / \mathrm{mL}$ chitosan inclusion (Table 
1). According to regression analysis, the levels of IL-1 and IL-2 tended to increase quadratically with increasing addition of chitosan $(\mathrm{P}=0.093$ and 0.106 , respectively). Although a similar trend was observed, there was no significant dosedependent relation for TNF- $\alpha$. The treatments of $80 \sim 160 \mu \mathrm{g} / \mathrm{mL}$ chitosan had higher levels of cytokines. However, the levels of cytokines in the culture fluid tended to decrease when the addition of chitosan in the medium increased to 320 $\mu \mathrm{g} / \mathrm{mL}$.

The levels of NO and iNOS in the culture fluid of all the treatments, except for the $320 \mu \mathrm{g} / \mathrm{mL}$ chitosan inclusion were higher than those of the control (Table 2). With increasing addition of chitosan, NO levels tended to increase quadratically ( $\mathrm{p}=0.053)$, which was highest when the medium contained $80 \mu \mathrm{g} / \mathrm{mL}$ chitosan. Consistent with the tendency of NO levels, iNOS levels also increased quadratically with increasing chitosan $(\mathrm{p}=0.157)$ and was highest at $80 \mu \mathrm{g} / \mathrm{mL}$ chitosan. However, the reduction of NO and iNOS levels occurred when chitosan supplementation was increased to $320 \mu \mathrm{g} / \mathrm{mL}$.

Compared with that in the control, iNOS mRNA expression in the chitosan treatments exhibited to be enhanced (Table 3). According to regression analysis, iNOS mRNA expression increased quadratically $(\mathrm{p}<0.05)$ with increasing addition of chitosan, which was highest when the chitosan supplementation was $80 \sim 160 \mu \mathrm{g} / \mathrm{mL}$. However, the reduction of iNOS mRNA expression occurred when chitosan supplementation was increased to $320 \mu \mathrm{g} / \mathrm{mL}$.

Table 1 - Effects of chitosan on levels of cytokines in culture fluid of peritoneal macrophages.

\begin{tabular}{lllllllll}
\hline \multirow{2}{*}{ Items } & \multicolumn{4}{c}{ Concentration of chitosan $(\boldsymbol{\mu g} / \mathbf{m L})$} & \multirow{2}{*}{ SEM $^{\mathbf{1}}$} & \multicolumn{2}{c}{$\boldsymbol{P}$-Value } \\
\cline { 2 - 5 } \cline { 4 - 6 } & $\mathbf{0}$ & $\mathbf{4 0}$ & $\mathbf{8 0}$ & $\mathbf{1 6 0}$ & $\mathbf{3 2 0}$ & & Linear & Quadratic \\
\hline $\mathrm{IL}^{2}{ }^{2}(\mathrm{ng} / \mathrm{mL})$ & 0.372 & 0.404 & 0.411 & 0.386 & 0.341 & 0.021 & 0.129 & 0.093 \\
$\mathrm{IL}^{3}{ }^{3}(\mathrm{ng} / \mathrm{mL})$ & 9.953 & 10.395 & 10.483 & 11.257 & 10.135 & 0.686 & 0.839 & 0.106 \\
$\mathrm{TNF}-\alpha^{4}(\mathrm{ng} / \mathrm{mL})$ & 4.648 & 5.189 & 5.330 & 5.229 & 4.840 & 0.270 & 0.922 & 0.221 \\
\hline
\end{tabular}

Note: ${ }^{1} \mathrm{SEM}=$ standard error of the mean. ${ }^{2} \mathrm{IL}-1=$ interleukin- $1,{ }^{3} \mathrm{IL}-2=$ interleukin- $2,{ }^{4} \mathrm{TNF}-\alpha=$ tumor necrosis factor- $\alpha$

Table 2 - Effects of chitosan on levels of NO and iNOS in culture fluid of peritoneal macrophages.

\begin{tabular}{lllllllll}
\hline \multirow{2}{*}{ Items } & \multicolumn{4}{c}{ Concentration of chitosan $(\boldsymbol{\mu g} / \mathbf{m L})$} & \multirow{2}{*}{ SEM $^{\mathbf{1}}$} & \multicolumn{2}{c}{$\boldsymbol{P}$-Value } \\
\cline { 2 - 5 } \cline { 5 - 6 } & $\mathbf{0}$ & $\mathbf{4 0}$ & $\mathbf{8 0}$ & $\mathbf{1 6 0}$ & $\mathbf{3 2 0}$ & & Linear & Quadratic \\
\hline $\mathrm{NO}^{2}(\mu \mathrm{mol} / \mathrm{L})$ & 72.12 & 72.48 & 74.69 & 72.99 & 67.30 & 1.835 & 0.137 & 0.053 \\
$\mathrm{iNOS}^{3}(\mathrm{U} / \mathrm{mL})$ & 35.80 & 36.25 & 39.83 & 35.68 & 33.64 & 1.587 & 0.170 & 0.157 \\
\hline
\end{tabular}

Note: ${ }^{1} \mathrm{SEM}=$ standard error of the mean. ${ }^{2} \mathrm{NO}=$ nitric oxide, ${ }^{3} \mathrm{iNOS}=$ inducible nitric oxide synthase

Table 3 - Effects of chitosan on iNOS mRNA expression in peritoneal macrophages $\left(2^{-\Delta \mathrm{Ct}}\right)$.

\begin{tabular}{|c|c|c|c|c|c|c|c|c|}
\hline \multirow[t]{2}{*}{ Items } & \multicolumn{5}{|c|}{ Concentration of chitosan $(\mathrm{mg} / \mathrm{kg})$} & \multirow{2}{*}{ SEM $^{1}$} & \multicolumn{2}{|c|}{ P-Value } \\
\hline & $\mathbf{0}$ & 40 & 80 & 160 & 320 & & Linear & Quadratic \\
\hline iNOS $^{2}$ & 0.0155 & 0.0244 & 0.0301 & 0.0417 & 0.0329 & 0.0048 & 0.926 & 0.003 \\
\hline
\end{tabular}

\section{DISCUSSION}

It is well known that chitosan is able to enhance the immune function. This is related to macrophage activation. Nishimura et al. (1986) and Peluso et al. (1994) have shown that chitosan can stimulate the macrophage and increase IL-1, IL-2and TNF- $\alpha$ secretion in the rats. Lim et al. (1997) also have shown the similar result that chitosan improved the immune functions by upregulating the TNF- $\alpha$, IL- 1 and M-CSF secretion in the macrophage of rats. The present study showed a dose-dependent tendency between chitosan supplement in the medium and the levels of IL-1, IL-2 and TNF- $\alpha$ in peritoneal macrophage culture fluid of broiler chickens. The treatments of $80 \sim 160 \mu \mathrm{g} / \mathrm{mL}$ chitosan had higher levels of cytokines. This suggested that chitosan had better effects on the secretory function of peritoneal macrophage, which was related to the supplemental dosage of chitosan in the medium. The mechanisms of chitosan stimulateing the secretory functions of peritoneal macrophage may be that the amino groups in the molecular structure 
of chitosan can be identified by the receptor on macrophages surface and activate the macrophages to a certain extent (Tokura et al. 1999). Activated macrophages secrete hundreds kinds of bioactive substances such as NO, IL- 1 , TNF- $\alpha$, IFN- $\gamma$, and reactive oxygen, many of which are related to the immune response and inflammation (Lin et al. 1996). Mei et al. (1994) studied in vivo and demonstrated the similar result that intraperitoneal injection of $2 \%$ chitosan could significantly improve IL-2 secretion of mouse spleen lymphocytes and increase spleen NK cell activity obviously. Chitosan could also increase IL-2 activity of spleen lymphocyte in vitro of broilers in a dose-dependent manner and $80 \mu \mathrm{g} / \mathrm{mL}$ chitosan had better effects (Shi 2004).

The effects of chitosan and other polysaccharides on NO and iNOS secretion and iNOS mRNA expression in mononuclear cells, especially macrophages were studied mainly in mice and other mammals such as human. Glycyrrhiza polysaccharides, Lentinan and polyporus polysaccharide could induced NO production by the murine peritoneal macrophages (Nose et al. 1998; Huang et al. 1999; Hou et al. 2000), and oligochitosan could significantly increase the activity of iNOS and induce the synthesis of NO in macrophages of the rats (Yu et al. 2004). Proteinbound polysaccharide also could promote the iNOS gene expression and increase the mRNA transcription and protein synthesis in the rats (Wang et al. 1999). Peluso et al. (1994) also demonstrated that chitosan could stimulate the macrophages in the rats and increase $\mathrm{NO}$ secretion. Porporatto et al. (2003) have shown the similar result that $0.05 \% \sim 0.1 \%$ chitosan significantly enhanced the content of $\mathrm{NO}$ and the expression of iNOS mRNA in rat macrophages. Shi (2004) also showed that chitosan supplementation had better effects on the splenocyte of broilers iNOS and NO production in dose-dependent manner, but its effect on iNOS gene expression had not been studied. The present study found that $80 \sim 160 \mu \mathrm{g} / \mathrm{mL}$ chitosan has better effects on the expression of iNOS mRNA and synthesis of iNOS and NO, and iNOS mRNA expression increased quadratically $(\mathrm{P}<0.05)$, NO and iNOS levels tended to increase quadratically with increasing addition of chitosan. But chitosan tended to decrease the levels of NO and iNOS in the medium and lessen iNOS expression at a higher level of inclusion ( $320 \mu \mathrm{g} / \mathrm{mL}$ medium).
This suggested that there was a threshold level of chitosan inclusion beyond which the reduction of iNOS expression in the macrophages of broiler chickens occurred. Therefore, the mechanism by which chitosan promoted NO production and enhanced the immune functions was probably that appropriate dosage of chitosan activated the iNOS mRNA expression and NO secretion.

\section{CONCLUSIONS}

From the results, it was concluded that one of the mechanisms by which chitosan stimulated the immune functions of broiler chickens could be chitosan enhancing iNOS activity and expression, increasing the production of NO, stimulating the macrophage and increasing the IL-1, IL-2 and TNF- $\alpha$ secretion, and finally resulting in the enhancement of immune functions of the chickens. But, there was a threshold level of chitosan inclusion beyond which progressive reductions in immune functions of broiler chickens would occur.

\section{ACKNOWLEDGMENTS}

The authors gratefully acknowledge the support of the National Natural Science Foundation of China (Project No. 30660130).

\section{REFERENCES}

Andus T, Rogler G, Daig R, Falk W, Scholmcrich J, Gross V. The role of macrophages. In: Tygat Ed GNJ, JFWM, Van Deventer SJH, editors. Inflammatory bowel disease. Netherlands: Kluwer Dordrecht; 1995. p. 281-297.

Ding AH. Release of reactive nitrogen intermediates and reactive oxygen intermediates from mouse peritoneal macrophages. J Immunol. 1988; 141 (7):2407-2412.

Hou G, Huang DN, Zhu QF. Effects of grifola polysaccharide on nitric oxide production in mouse peritoneal macrophages and its mechanism. Chinese Geraeol. 2000; 20:233-235.

Huang DN, Hou G, Zhu QF. Effects of lentinan on nitric oxide production in mouse peritoneal macrophages and its mechanism. Chinese J Basic Med Sci Clin. 1999; 19:43-47.

Jamas S, Easson D, Stroff GRO. Method for producing soluble glucan. United States Patents, Patent NO. 5633369. 1997. 
Korhonen R, Lahti A, Kankaanranta H, Moilanen E. Nitric oxide production and signaling in inflammation. Curr Drug Targets Inflamm Allergy. 2005; 4:471-479.

Li HY, Yan SM, Shi BL, Guo XY. Effect of chitosan on nitric oxide content and inducible nitric oxide synthase activity in serum and expression of inducible nitric oxide synthase mRNA in small intestine of broiler chickens. Asian-Aust J Anim Sci. 2009; 22 (7): 1048-1053.

Li P, YinYL, Li D, Kim SW, Wu G. Amino acids and immune function. Br J Nutr. 2007; 98:237-252.

Lim BO, Yamada K, Nonaka M, Kuramoto U, Hung P, Sugano M. Dietary fibers modulate indices of intestinal immune function in rats. J Nutr. 1997; 127:663-667.

Lin AW, Chang CC, Mccormick CC. Molecular cloning and expression of an avian macrophage nitric-oxide synthase cDNA and the analysis of the genomic 5'-anking region. J Biol Chem. 1996; 271:11911-11919.

Liu WK, Ng TB, Sze SF, Tsui KW. Activation of peritoneal macrophages by polysaccharo-peptide from the mushroom, coriolus versicolor. Immunopharm. 1993; 26:139-146.

Mei XW, Pang BS, Liu EQ. Effects of chitosan on mouse spleen NK cell activity and IL-2 secretion. Chinese J Capital Med College. 1994; 15:141-143.

Moilanen E, Whittle B, Moncada S. Nitric oxide as a factor in inflammation. In: Gallin EdJI, Snyderman R, editors. Basic Principles and Clinical Correlates. Philadelphia: Lippincott Williams \& Wilkins;1999.p. 787-800.

Nishimura K, Nishimura S, Seo H, Nishi N, Tokura S, Azuma I. Macrophage activation with multi-porous beads prepared from partially deacetylated chitin. $J$ Biomed Mater Res. 1986; 20:1359-1372.

Nose M, Terawaki K, Oguri K, Ogihara Y, Yoshimatsu K, Shimomura K. Activation of macrophages by crude polysaccharide fractions obtained from shoots of Glycyrrhiza glabra and hairy roots of Glycyrrhiza uralensis in vitro. Biol Pharm Bull. 1998; 21:11101102.

Peluso G, Petillo O, Ranieri M, Santin M, Ambrosio L, Calabro D, et al. Chitosan-mediated stimulation of macrophage function. Biomaterials. 1994; 15:12151220.
Porporatto C, Bianco ID, Riera CM, Corres SG. Chitosan induces different L-arginine metabolic pathways in resting and inflammatory macrophages. $J$ Biochem Biophys Res Commun. 2003; 304:266-272.

Qureshi MA. Avian macrophage and immune response: an overview. Poult Sci. 2003; 82:691-698.

Shi BL. Effects of chitosan on growth performance and immune function in broilers and the underlying mechanisms[PhD Thesis]. Beijing, China: China Agricultural University; 2004.

Shi BL, Li DF, Piao XS. Effects of chitosan on growth performance and immune function in broilers. Chinese J Anim Sci. 2005; 41(1):9-11.

Tokura S, Tamura H, Azuma I. Immunological aspects of chitin and chitin derivatives administered to animals. EXS. 1999; 87:279-292.

Tropea A, Tiberi F, Minici F, Orlando M, Gangale MF, Romani F, et al. Ghrelin affects the release of luteolytic and luteotropic factors in human luteal cells. J Clin Endocrinol Metab. 2007; 92:3239-3245.

Wang JW, Chen Y, Zhou M. Effect of protein-bound polysaccharide on inhibition of LDL oxidation induced by macrophages and their relation with iNOS gene expression. Chinese J First Milit Med Univ. 1999; 19:25-28.

Wang W, Inoue N, Nakayama T. An assay method for nitric oxide synthase in crude samples by determining product $\mathrm{NADP}^{+}$. Anal Biochem. 1995; 227 (2): 274280.

Xing Z, Schat KA. Inhibitory effects of nitric oxide and gamma interferon on in vitro and in vivo replication of Marek's disease virus. J Virol. 2000; 74:36053612.

Yu Z, Zhao L, Ke H. Potential role of nuclear factorkappa $\mathrm{B}$ in the induction of nitric oxide and tumor necrosis factor-alpha by oligochitosan in macrophages. Int Immunopharmacol. 2004; 4:193200.

Zhang M, Zhang LH, Ye DJ. Change of the level of serum cytokines in patients with epilepsy after cerebral infarction. J Clin Neurol. 2008; 21(2):103104.

Received: April 11, 2013; Accepted: November 04, 2013. 